\title{
Estado Actual de las Investigaciones Tafonómicas Naturalistas en Punta Entrada y Monte León (Patagonia Meridional)
}

\author{
Sebastián Muñoz e Isabel Cruzii
}

\section{RESUMEN}

Se presentan los resultados de las investigaciones tafonómicas naturalistas realizadas desde 2005 en la desembocadura del río Santa Cruz y el parque Nacional Monte León (Patagonia Meridional) dirigidas a conocer los modos en que tiene lugar el reciclado de restos de vertebrados en un mismo contexto ambiental, la estepa costera patagónica. Se resume el conocimiento generado sobre: el tiempo involucrado en la desarticulación de carcasas de vertebrados; los procesos y actores intervinientes y las características de los conjuntos así formados. Los resultados sugieren que la desarticulación y la dispersión están condicionadas principalmente por los procesos vinculados con la descomposición de los tejidos blandos. Esto implica un lento reciclado de las carcasas y afecta la disponibilidad de huesos con posibilidades de integrar registro fósil generando huesos que están más tiempo expuestos pero que se encuentran escasamente dispersos.

Palabras Clave:Tafonomía Naturalista,Vertebrados, Patagonia Meridional

\section{ABSTRACT}

Naturalistic taphonomic research undertaken since 2005 in Santa Cruz river mouth and Monte León National Park (Southern Patagonia, Argentina) is presented. The research is aimed to explore vertebrate bone recycling under the same environmental conditions, the coastal patagonian steppe. Three aspects are considered: time involved in vertebrate carcass disarticulation; the taphomic processes and actors involved and the main features of the resulting assemblages. Disarticulation rate and bone scattering is driven by decomposition related processes. This may imply a slow bone recycling and may condition bones available for becoming part of the fossil record by generating disperse less bones exposed for a considerable time span.

Key Words: Naturalistic Taphonomy, Vertebrates, Patagonia Meridional

\footnotetext{
${ }^{i}$ LaZTA-IDACOR, CONICET-Facultad de Filosofía y Humanidades,

Universidad Nacional de Córdoba. Correo-e: smunoz@conicet.gov.ar

ii Unidad Académica Río Gallegos, Universidad Nacional de la Patagonia

Austral. Correo-e: isabelzooarqueologia@gmail.com
}

Recibido: 03-12-2012 - Revisado: 02-05-2013 - Aceptado: 10-10-2013 


\section{INTRODUCCIÓN}

En este trabajo se presenta el estado actual de las investigaciones tafonómicas que se desarrollan desde el año 2005 en la desembocadura del río Santa Cruz y el Parque Nacional Monte León (Patagonia Meridional,Argentina) y que están dirigidas a estudiar la variabilidad potencial que ofrece el continuo natural-cultural de conjuntos óseos de vertebrados depositados en la costa patagónica. Aplicamos una perspectiva tafonómica naturalista para entender el reciclado de restos de vertebrados en un mismo contexto ambiental, la estepa costera del sur del continente, caracterizada por un clima templado y seco con temperaturas medias anuales en el orden de los $4^{\circ}-8^{\circ} \mathrm{C}$ y una precipitación media anual de entre 100 y $200 \mathrm{~mm}$. Aquí abordamos tres aspectos vinculados con la formación de asociaciones de huesos naturales: a) el tiempo involucrado en la desarticulación de las carcasas de vertebrados, b) los procesos y actores actuantes y c) las características de las dispersiones de huesos resultantes. Consideramos tres vertebrados terrestres: guanaco (Lama guanicoe), zorro (Pseudalopex culpaeus) y ñandú (Rhea pennata) y uno marino, el lobo marino común (Otaria flavescens). Las observaciones se realizaron en intervalos de 4 a 12 meses, cubriendo un mínimo de 3 años y medio y un máximo de 6 años y medio, según el momento de depositación o primera observación de cada carcasa.

En un trabajo previo (Cruz y Muñoz 2010) analizamos las trayectorias de desarticulación y dispersión de una de las carcasas de guanaco y la de zorro durante tres años desde la muerte de los individuos y registramos que en ese lapso las mismas presentaron diferencias importantes en el ritmo de adquisición de modificaciones. En un año la desarticulación de la carcasa de guanaco recién comenzaba mientras que la de zorro progresó hasta involucrar gran parte de las unidades anatómicas. Concluimos que los factores climáticos fueron los que tuvieron una mayor importancia en la desarticulación, mientras que el pisoteo animal probablemente haya sido la causa más importante de la dispersión, en un contexto en que no se registró acción de carnívoros (Cruz y Muñoz 20 I0). Aquí ampliamos las observaciones sobre esas dos carcasas y aplicamos la misma metodología, basada en estadios amplios para analizar la desarticulación y dispersión (ver Tabla I), a una diversidad de vertebrados de distintos tamaños, hábitos de vida y estructuras anatómicas registrados en diferentes ambientes de depositación (ver Tablas 2 y 3$)$.

\begin{tabular}{|l|l|l|}
\hline & Desarticulación & Dispersión \\
\hline Nula & Todos los huesos articulados & carcasa articulada o con escasa desarticulación \\
\hline Inicial & Desarticulación de cráneo y algunas extremidades & $\begin{array}{l}\text { movimiento de elementos o porciones articuladas fuera de su posición } \\
\text { anatómica pero en el lugar de depositación de la carcasa }\end{array}$ \\
\hline Avanzada & $\begin{array}{l}\text { Desarticulación de costillas, elementos de las } \\
\text { extremidades, posiblemente la mandibula }\end{array}$ & $\begin{array}{l}\text { Desplazamiento de varios metros. Se observa un núcleo central con } \\
\text { mayor cantidad de huesos }\end{array}$ \\
\hline Terminal & Desarticulación de columna vertebral & Núcleo central de depositación sin concentración de huesos. \\
\hline Completa & No quedan unidades articuladas & \\
\hline
\end{tabular}

Tabla 1: Estadios considerados para describir y analizar la desarticulación y dispersión de las carcasas estudiadas. Modificada de Cruz y Muñoz (2010) y bibliografía allí citada.

Table 1: Stages applied to carcass dismembering and dispersion. Modified from Cruz and Muñoz (2010) and references therein. 


\begin{tabular}{|c|c|c|c|c|c|}
\hline & Localidad & Ambiente & $\begin{array}{c}\text { Primera } \\
\text { observación }\end{array}$ & Estado & \begin{tabular}{|c|} 
Total \\
registros
\end{tabular} \\
\hline \multicolumn{6}{|l|}{ Guanaco } \\
\hline $\begin{array}{l}\text { Carcasa I } \\
(\mathrm{GCl})\end{array}$ & $\begin{array}{l}\text { Punta } \\
\text { Entrada }\end{array}$ & $\begin{array}{l}\text { Médano } \\
\text { inactivo }\end{array}$ & $\begin{array}{l}\text { Noviembre } \\
2005\end{array}$ & $\begin{array}{l}\text { Completo, articulado, } \\
\text { fresco }\end{array}$ & 11 \\
\hline $\begin{array}{l}\text { Carcasa } 3 \\
\text { (GC3) }\end{array}$ & \begin{tabular}{|l} 
Punta \\
Entrada
\end{tabular} & $\begin{array}{l}\text { Médano } \\
\text { inactivo }\end{array}$ & $\begin{array}{l}\text { Noviembre } \\
2009\end{array}$ & $\begin{array}{l}\text { Completo, articulado, } \\
\text { fresco }\end{array}$ & 4 \\
\hline $\begin{array}{l}\text { Carcasa } \\
(\mathrm{GCMLI})\end{array}$ & $\begin{array}{l}\text { Monte } \\
\text { León }\end{array}$ & $\begin{array}{l}\text { Fondo de } \\
\text { cañadón }\end{array}$ & $\begin{array}{l}\text { Noviembre } \\
2008\end{array}$ & $\begin{array}{l}\text { Completo } \\
\text { Desarticulación } \\
\text { inicial, tejidos blandos } \\
\text { presentes }\end{array}$ & 7 \\
\hline Zorro & $\begin{array}{l}\text { Punta } \\
\text { Entrada }\end{array}$ & $\begin{array}{l}\text { Médano } \\
\text { inactivo }\end{array}$ & $\begin{array}{l}\text { Noviembre } \\
2005\end{array}$ & $\begin{array}{l}\text { Completo, articulado, } \\
\text { fresco }\end{array}$ & 10 \\
\hline Ñandú & $\begin{array}{l}\text { Punta } \\
\text { Entrada }\end{array}$ & $\begin{array}{l}\text { Médano } \\
\text { activo }\end{array}$ & $\begin{array}{l}\text { Noviembre } \\
2008\end{array}$ & $\begin{array}{l}\text { Incompleto, articulado, } \\
\text { seco }\end{array}$ & 5 \\
\hline \multicolumn{6}{|l|}{$\begin{array}{l}\text { Lobo } \\
\text { Marino }\end{array}$} \\
\hline $\begin{array}{l}\text { Carcasa } 3 \\
(\text { LC3) }\end{array}$ & $\begin{array}{l}\text { Punta } \\
\text { Entrada }\end{array}$ & $\begin{array}{l}\text { Cordón } \\
\text { litoral o } \\
\text { berma }\end{array}$ & $\begin{array}{l}\text { Septiembre } \\
2009\end{array}$ & $\begin{array}{l}\text { Completo, articulado, } \\
\text { fresco }\end{array}$ & 5 \\
\hline $\begin{array}{l}\text { Carcasa } 4 \\
(\mathrm{LC} 4)\end{array}$ & $\begin{array}{l}\text { Punta } \\
\text { Entrada }\end{array}$ & $\begin{array}{l}\text { Cordón } \\
\text { litoral o } \\
\text { berma }\end{array}$ & $\begin{array}{l}\text { Septiembre } \\
2009\end{array}$ & $\begin{array}{l}\text { Completo, articulado, } \\
\text { seco }\end{array}$ & 5 \\
\hline
\end{tabular}

Tabla 2: Procedencia, ambiente de depositación, condición y fecha al momento del primer registro y cantidad de observaciones efectuadas en las carcasas consideradas en este estudio.

Table 2: Provencience, depositional environment, condition and date at initial record and number of records taken at each carcass considered in this study.

\begin{tabular}{|c|c|c|c|c|c|c|c|}
\hline & \multirow{2}{*}{ Zorro } & \multirow{2}{*}{ Ñandú } & Guanaco & \multirow{2}{*}{$\begin{array}{l}\text { Lobo Marino } \\
\text { GC3 }\end{array}$} & \multirow[b]{2}{*}{ GCMLI } & \multirow[b]{2}{*}{ LC3 } & \multirow[b]{2}{*}{ LC4 } \\
\hline & & & $\mathrm{GCl}$ & & & & \\
\hline Articulada & 6 meses & $s / d$ & I año & \multirow[t]{2}{*}{6 meses } & $s / d$ & \multirow[t]{2}{*}{2 años } & \multirow[t]{2}{*}{$s / d$} \\
\hline \multicolumn{5}{|l|}{ Desarticulación } & & & \\
\hline Inicial & 6 meses & I año & I,5 años & \multirow[b]{2}{*}{2 años } & + de 6 meses & En curso & + de 2 años \\
\hline Avanzada & $s / d$ & 2 años & I año & & I año & No & En curso \\
\hline Terminal & 5 años & I año & $\begin{array}{l}3 \text { años, en } \\
\text { curso }\end{array}$ & $\begin{array}{l}6 \text { meses, en } \\
\text { curso }\end{array}$ & I, 5 años, en curso & No & No \\
\hline Completa & En curso & $S / d$ & No & & No & No & No \\
\hline \multicolumn{8}{|l|}{ Dispersión } \\
\hline Nula & 6 meses & $\begin{array}{l}+ \text { de I } \\
\text { año }\end{array}$ & I año & 6 meses & $s / d$ & 2 años & $s / d$ \\
\hline Inicial & $s / d$ & \multirow{3}{*}{ 2,5 años } & 6 meses & + I año & I año & En curso & 2 años \\
\hline Avanzada & + 4 años & & $\begin{array}{l}4 \text { años, en } \\
\text { curso }\end{array}$ & I año, en curso & 2,5 años, en curso & No & En curso \\
\hline Completa & En curso & & No & No & No & No & No \\
\hline Tiempo total & 6,5 años & 3,5 años & 6,5 años & 2,5 años & 3,5 años & 2,5 años & 2,5 años \\
\hline
\end{tabular}

Tabla 3: Duración de las etapas de desarticulación y dispersión registradas en cada una de las carcasas estudiadas. Tiempo total en el que se realizaron las observaciones. $\mathrm{S} / \mathrm{D}=$ sin datos registrados.

Table 3: Lenght of disarticulation and dispersión stages recorded for each carcass. Total time elapsed for recording. $S / D=n o$ data taken 


\section{RESULTADOS}

Tiempo involucrado en la desarticulación

En Punta Entrada las carcasas de guanaco se encontraron depositadas sobre los sedimentos eólicos a diferentes distancias de la línea de costa actual, mientras que en Monte León está ubicada en el fondo de un cañadón. En las dos localidades éstas alcanzaron una desarticulación inicial dentro de los 6-12 meses desde la depositación. El paso a una desarticulación avanzada tomó al menos un año y medio y se vincula con la velocidad de pérdida de tejidos blandos (ver más adelante). Una vez alcanzada la desarticulación avanzada el estadio siguiente terminal - se desarrolló lentamente, pudiendo tomar más de 3 años. Este estadio involucró la desvinculación de las articulaciones cartilaginosas secundarias (vértebras torácicas, lumbares, etc.), las vértebras individuales, así como pelvis y sacro, con excepción de las vértebras caudales que permanecieron articuladas, incluso 5 años y medio después de la depositación. No se registraron casos de desarticulación completa.

Si bien la muestra de los vertebrados terrestres de menor tamaño (zorro y ñandú) es más limitada que la de guanaco, lo cual es esperable ya que se trata de una investigación naturalista, los resultados alcanzados muestran algunas diferencias que pueden considerarse valiosas a nivel exploratorio. En efecto, una vez alcanzada la desarticulación terminal, aproximadamente a los 2 años de depositación, la carcasa de zorro permaneció en este estadio por otros 5 años, tras lo cual se inició la desarticulación completa, etapa actualmente en curso. La carcasa de ñandú, en cambio, permaneció un año en la etapa inicial, con la mayoría de las articulaciones sinoviales y cartilaginosas sin modificación, dos años en la etapa avanzada y un año en el estadio terminal, lo cual es inusual en relación a lo registrado en otros sectores del sur de Patagonia, donde la desarticulación es más rápida (Cruz 2003). Es decir, los vertebrados de menor tamaño completaron la desarticulación terminal antes que las carcasas de guanaco, aunque en ritmos diferentes. Las diferencias tienen que ver con la duración del estadio avanzado. Mientras que las carcasas de guanaco ocuparon aproximadamente un año en este estadio, la de zorro fue muy breve, y no pudo registrarse de manera detallada, y la de ñandú fue extensa ( 2 años).
En lo que hace a las carcasas de lobo marino, la primera pérdida de conexión anatómica requirió un mínimo de 2 años (LC3, separación del cráneo y mandíbula). De este modo dio comienzo la desarticulación inicial, permaneciendo con muy poco cambios desde momento. La carcasa CL4, que estaba en estado inicial al momento del primer registro, permaneció así por más de 2 años y actualmente está en la etapa avanzada, con el atlas-cráneo, húmeroescápula y las distintas vértebras desarticuladas, permaneciendo la pelvis articulada al sacro y las lumbares. Se observa, así que la desarticulación de las carcasas de éstos mamíferos marinos ocupa más tiempo que las de los vertebrados terrestres.

Procesos y actores involucrados en la desarticulación:

En la estepa patagónica los actores tafonómicos tienen poco impacto en la destrucción de huesos y su acción sobre los tejidos blandos también es escasa (Borrero 1988, Cruz 2003, Cruz y Muñoz 2010). En un contexto de este tipo el ritmo descomposición de éstos últimos es un proceso que adquiere particular importancia para dar cuenta de la desarticulación (Lyman 1994). En este sentido podemos señalar que las carcasas de guanaco permanecieron con cuero entre 2 y 3 años, lapso durante el cual parte de los tejidos blandos primero se secaron y luego desaparecieron. Esto hizo que por lapsos considerables hubiese poca pérdida y dispersión de huesos. A medida que desaparecía el tejido blando, las primeras articulaciones que se separaron fueron las sinoviales, es decir aquellas que permiten el libre movimiento entre los elementos articulados, principalmente la articulación de la pelvis con el fémur, el fémur con la tibia y la tibia con los metatarsos. Algo semejante ocurrió con la escápula y el húmero y éste último con el radioulna. Es decir, en todos los casos la pérdida de este tejido aceleró el paso hacia una concentración de huesos más o menos articulados, según el contexto de depositación.

En lo que respecta a las carcasas de lobo marino, durante los 2 años y medio se registró el desecamiento del cuero y la pérdida parcial de tejidos blandos, que se endurecieron, impidiendo le pérdida de conexión anatómica en gran parte de la anatomía del animal. Esta integridad anatómica fue mayor en la carcasa CL3, que se encontraba fresca 
al momento de depositación, y menor en la carcasa CL4 que se encontraba seca. A diferencia de lo que ocurre con los guanacos, tras 2 años y medio ambas carcasas siguen conservando una gran cantidad de cuero y muchos elementos bien articulados, como por ejemplo los de las aletas.

La carcasa de zorro permaneció con abundante tejido blando durante un año, conservándolo exclusivamente sobre las extremidades y la cola a partir del año y medio (Cruz y Muñoz 2010). A los tres años estos tejidos estaban prácticamente desaparecidos. La carcasa de ñandú, por su parte, tenía escasos tejidos blandos al momento del primer registro, principalmente plumas y fibras, que mantuvieron articulados los distintos elementos óseos por un tiempo prolongado.

En síntesis, el proceso de descomposición tiene un papel importante en el desarrollo de la secuencia de desarticulación. El tiempo en que se desarrolla este proceso depende fundamentalmente de que exista un umbral de temperatura adecuado (mayor a $15^{\circ} \mathrm{C}$ ) para que se produzcan las reacciones químicas necesarias para ello. Las bajas temperaturas en la zona de estudio tienen como consecuencia una escasa actividad microbiana y una mayor permanencia en el tiempo de las partes anatómicas articuladas. A esto se suman las características anatómicas de cada taxón, particularmente la rigidez relativa de las distintas articulaciones del esqueleto y los atributos morfométricos y físicos de los huesos, que condicionarán su trayectoria como partículas sedimentarías una vez independizados de la carcasa (Lyman 1994).

Características de las dispersiones de huesos:

La dispersión fue nula para las carcasas de vertebrados terrestres dentro de los primeros 6-12 meses de observación. Las distintas carcasas de guanaco alcanzaron una dispersión inicial en un lapso de tiempo similar, no siendo posible registrar esta etapa en la carcasa de zorro ya que cambió de estado hacia una dispersión avanzada ocurrió entre dos observaciones, es decir 6 meses (Cruz y Muñoz 2010). Aunque no fue posible registrar las distintas etapas en la carcasa de ñandú registramos que, tras un período comparativamente prolongado sin dispersión (más de I año), en los 2 años y medio siguientes se produjeron todas las etapas restantes (inicial, avanzada y completa). Las carcasas de zorro y guanaco, en cambio, permanecieron como dispersiones avanzadas por más de cuatro años, habiendo iniciado la primera de ellas la etapa de dispersión completa. Las de lobo marino muestran una secuencia más lenta. La carcasa CL3 permaneció sin dispersión dos años y está actualmente en desarrollo la dispersión inicial. La CL4, permaneció en estadio inicial por dos años y recientemente ha iniciado la dispersión avanzada.

\section{DISCUSIÓN}

El estudio de la desarticulación y dispersión de carcasas animales tiene como objetivo conocer el ritmo que imponen y el tiempo que involucran estos procesos respecto de la formación de acumulaciones naturales de huesos, así como evaluar los principales actores y procesos que las causan. De esta manera se puede abordar el aporte natural de huesos al paisaje, las posibilidades potenciales de formación de acumulaciones y la firma fósil que las caracteriza. A diferencia de lo que ocurre en ambientes africanos en los que se han realizado los estudios tafonómicos naturalistas que usualmente utilizamos como referencia (por ej. Hill y Behrensmeyer 1984), en Patagonia los actores tafonómicos actuantes sobre los tejidos blandos y el cuero, predadores y carroñeros, son relativamente pocos (Borrero 1988, Cruz 2008; entre otros). Los resultados presentados son concordantes con estos antecedentes y muestran que la desarticulación está condicionada por la falta de un umbral de temperatura adecuado (mayor a $15^{\circ} \mathrm{C}$ ) y en consecuencia a una escasa actividad microbiana. Bajo estas condiciones la desarticulación produce asociaciones de restos que se dispersan sin que exista una interfase de transporte importante por lo que resultan de procesos de acumulación pasivos (sensu Badgley 1986, en Lyman 1994), lo que implica pocos huesos que se incorporan al potencial registro fósil, principalmente por fuerzas vinculadas con la gravedad, la acción del agua y/o el pisoteo animal. Este aporte podría resultar, entonces, en asociaciones de diferente tamaño que en el largo plazo reflejen eventos de depositación distintos y que, por lo tanto, tendrían escasa resolución temporal.

En términos generales, en esta zona de estudio se pudo registrar que la desarticulación de las carcasas de lobos marinos es más lenta 
que la de los vertebrados terrestres y que la dispersión avanzada es el estadio de duración más prolongada para el zorro y el guanaco. Estas relaciones confirman la importancia que tienen los tejidos blandos para mantener la organización anatómica y retardar la dispersión de restos óseos en el espacio, proceso que una vez iniciado puede llevar a la dispersión de huesos con un núcleo central relativamente estable. De esta manera, la resistencia del cuero y la rigidez relativa de las distintas articulaciones se encuentran entre los factores que condicionan fuertemente la secuencia y velocidad de desarticulación de las carcasas. Esto es distinto, por ejemplo, de lo informado para latitudes semejantes, como el mar del Norte (Schafer 1972), en donde en tan sólo 2 meses se registró la desorganización completa de una carcasa de pinnípedo principalmente por la acción de las lluvias que facilitan la desarticulación.

Por otro lado, si comparamos con contextos ecológicos de mayor competencia entre carnívoros, como Amboseli, Kenia, encontramos que la secuencia de desarticulación del guanaco se diferencia parcialmente respecto de ungulados de tamaño parecido como el topi (Damaliscus korrigum) y de menor tamaño como la gacela (Nanger granti). En el Gráfico I se comparan las articulaciones que pierden conexión tempranamente. Se aprecia que los dos guanacos de Punta Entrada y Monte León desarticularon más tempranamente los miembros traseros y los metapodios de las falanges. Un aspecto a explorar en el futuro es si estas diferencias están indicando una característica de la descomposición de las carcasas en la costa del sur de Patagonia que pueda resultar en un lento reciclado de huesos, como consecuencia de su exposición prolongada y escasa desarticulación.

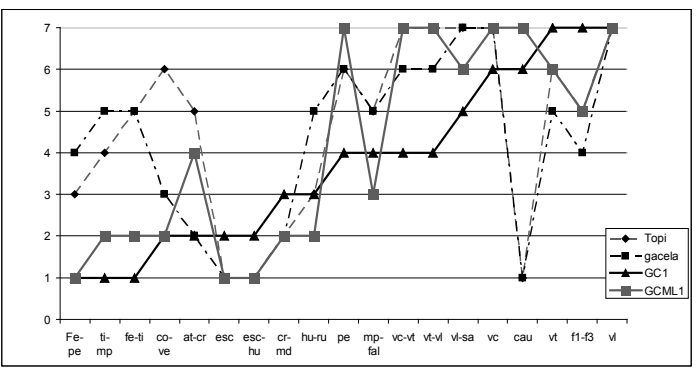

Figura 1: comparación de la secuencia de desarticulación del topi (Damaliscus korrigum), la gacela (Nanger granti) y dos guanacos (Lama guanicoe) considerados en este estudio. El eje de las abscisas se listan las articulaciones y en el de las ordenadas el orden de desarticulación, donde 1 es primero y 7 último. Datos tomados de Lyman 1994: Tabla 5.5.

Figure 1: comparison between disarticulation sequences of topi (Damaliscus korrigum), gazelle (Nanger granti) and two guanacos (Lama guanicoe) considered in this study. Main bone joints are presented on the $x$ axis and disarticulation order on the $y$-axis, being 1 the first and 7 the latter. Data taken from Lyman 1994: Table 5.5

Finalmente, los resultados obtenidos sugieren la importancia que la preservación diferencial de huesos articulados puede tener para entender las asociaciones naturales de huesos. Por ejemplo, la preservación de restos correspondientes a las desarticulaciones tempranas puede informar sobre sectores con potencial de enterramiento rápido, por ejemplo el fémur - pelvis en guanaco y zorro y cráneo y atlas para el lobo marino. El hallazgo de huesos aislados vinculados a desarticulaciones tardías, en cambio, sólo sería diagnóstico para algunos taxones como el guanaco y no en el caso del zorro,ya que este taxón evidencia una rápida desarticulación $y$, por lo tanto, sus huesos serían menos informativos de la dinámica de depositación y enterramiento. Estas expectativas y otras similares podrán discutirse en una próxima etapa de la investigación cuando se sumen al análisis un análisis detallado de los procesos vinculados con la gravedad (sensu Lyman 1994), que permitan evaluar el modo en que las características reseñadas para los procesos aquí analizados afectan diferencialmente la disponibilidad de los distintos elementos óseos para el enterramiento, así como la información sedimentológica de los loci de depositación. 


\section{AGRADECIMIENTOS:}

Financiamiento: CONICET/PIP II 2 20I20। 00359, UNPA 29A/302 y UNC SeCyT 162/12. Agradecemos al Sr. Víctor López de la Ea. Monte Entrance. La Municipalidad de Puerto Santa Cruz y el Parque Nacional Monte León asistieron en la logística de campo. Claudia Aguilar, Patricia Lobbia, Carolina Moreno, Adriana Pretto y Gustavo Nauto colaboraron en dichas tareas.

\section{BIBLIOGRAFÍA}

Borrero L.A. 1988. "Estudios tafonómicos en Tierra del Fuego: su relevancia para entender procesos de formación del registro arqueológico". En Arqueología Contemporánea Argentina, editado por H. Yacobaccio, pp. I3-32. Ediciones Búsqueda, Buenos Aires.
Cruz, I. 2003. Paisajes tafonómicos de restos de Aves en el sur de Patagonia continental. Aportes para la interpretación de conjuntos avifaunísticos en registros arqueológicos del Holoceno. Tesis Doctoral, Facultad de Filosofía y Letras, Universidad de Buenos Aires. MS

-.-- 2008. "Avian and Mammalian Bone Taphonomy in Southern Continental Patagonia. A Comparative Approach". Quaternary International 180:30-37.

Cruz, I. y A.S. Muñoz 2010. "Tafonomía comparativa: seguimiento de carcasas de mamíferos en Punta Entrada (Santa Cruz, Argentina)". En Zooarqueología a principios del siglo XXI. Aportes teóricos, metodológicos y casos de estudio, editado por M.A.Gutiérrez, M. De Nigris, P.M. Fernández, M. Giardina, A. Gil,A. Izeta, G. Neme y H.Yacobaccio, pp. 387-396. Ediciones del Espinillo, Buenos Aires.

Hill, A. y A.K. Behrensmeyer, 1984. "Disarticulation patterns of some modern East African Mammals". Paleobiology 10:366-376.

Lyman, R.1994. Vertebrate Taphonomy. Cambridge University Press, Cambridge.

Schafer, W. 1972. Ecology and Palaeoecology of Marine Environments. Chicago University Press, Chicago. 